\title{
Molecular hydrogen diffusion in nanostructured amorphous silicon thin films
}

\author{
Fatiha Kail, * Jordi Farjas, and Pere Roura \\ GRMT, Department of Physics, University of Girona, Campus Montilivi, Edif. PII, E17071 Girona, Catalonia, Spain \\ Pere Roca i Cabarrocas \\ LPICM, CNRS, Ecole Polytechnique, 91128 Palaiseau, France
}

(Received 3 June 2009; published 17 August 2009)

\begin{abstract}
We study hydrogen stability and its evolution during thermal annealing in nanostructured amorphous silicon thin films. From the simultaneous measurement of heat and hydrogen desorption, we obtain the experimental evidence of molecular diffusion in these materials. In addition, we introduce a simple diffusion model which shows good agreement with the experimental data.
\end{abstract}

DOI: $10.1103 /$ PhysRevB.80.073202

PACS number(s): 66.30.-h, 61.43.Dq, 68.60.Dv, 68.43.Vx

The use of hydrogenated amorphous silicon (a-Si:H) as the active layer in devices such as thin-film transistors and solar cells is possible, thanks to the defect passivation role of hydrogen $(\mathrm{H}) .{ }^{1}$ However, $\mathrm{H}$ is also involved in the degradation of the electronic properties of a-Si:H when exposed to light. ${ }^{2,3}$ In contrast, polymorphous silicon (pm-Si:H), a grade of nanostructured amorphous silicon, exhibits enhanced hole mobility ${ }^{4}$ and a lower density of metastable electronic defects after prolonged illumination., ${ }^{5,6}$ Despite the importance of $\mathrm{H}$ stability and evolution in a- $\mathrm{Si}: \mathrm{H}$ and $\mathrm{pm}-\mathrm{Si}: \mathrm{H}$, there is still a lot we do not know about it.

Evolved gas analysis (EGA) experiments are extensively used to analyze $\mathrm{H}$ stability and evolution. ${ }^{7}$ Samples are usually heated at a constant rate in vacuum and the desorbing $\mathrm{H}_{2}$ molecules are detected by mass spectrometry. For devicequality a-Si:H thin films, a single peak is observed at high temperatures $\left(500-700{ }^{\circ} \mathrm{C}\right) .{ }^{8}$ This is associated with $\mathrm{H}$ atoms that are dissolved in the Si-Si network, diffuse in atomic form and eventually reach the material's external surface where they form the $\mathrm{H}_{2}$ molecule and evolve. For lowquality films, with an interconnected void network, a lowtemperature peak $\left(300-400{ }^{\circ} \mathrm{C}\right)$ (Refs. 7 and 8) is also observed. This is attributable to the rupture of $\mathrm{Si}-\mathrm{H}$ groups located on the surfaces of internal voids and the simultaneous formation of $\mathrm{H}_{2}$ molecules that evolve without any significant delay.

We recently suggested, following EGA and DSC (differential scanning calorimetry) measurements, ${ }^{9,10}$ that the main dehydrogenation process in $\mathrm{pm}-\mathrm{Si}: \mathrm{H}$ has an intermediate character; it involves the formation of a $\mathrm{H}_{2}$ molecule that diffuses through the film. This process is due to $\mathrm{Si}-\mathrm{H}$ groups presumably located at the interfaces between the amorphous matrix and the embedded nanocrystals.

The EGA thermogram of Fig. 1 shows that this process has a characteristic two-peak structure: a sharp peak (P1) followed, at a higher temperature, by a broader peak (P2). What is extremely puzzling is the fact that whereas peak P1 is associated with an exothermic peak of the DSC curve, there is no calorimetric counterpart associated with peak P2. This is the case despite the number of $\mathrm{H}$ atoms evolving with peak P2 (the area below the EGA curve) being much higher than the number evolving with peak P1 (Fig. 1). The absence of an exothermic peak P2 is even more obvious with a thicker layer ${ }^{9}$ (inset of Fig. 1). In this case, the results ob- tained at two heating rates make the common identification of peak P1 in the DSC and EGA thermograms clearer. Since the calorimetric signal is due to residual oxidation occurring during the breaking and rearranging of bonds, ${ }^{12}$ we suggest that the $\mathrm{Si}-\mathrm{H}$ groups dissociate at peak $\mathrm{P} 1$ with simultaneous formation of $\mathrm{H}_{2}$ molecules and that most of these molecules evolve later (at peak P2) due to diffusion. We would like to point out that this interpretation of peak P1 is strengthened by the fact that its thermal activation energy is very similar to that observed for other $\mathrm{Si}-\mathrm{H}$ groups ${ }^{9}$ [polymeric $\left(\mathrm{Si}-\mathrm{H}_{2}\right)_{n}$ and $\mathrm{H}$ on the surfaces of voids] for which the simultaneous formation of $\mathrm{H}_{2}$ has been demonstrated by isotope-labeling experiments. ${ }^{8}$ Furthermore, other $\mathrm{Si}-\mathrm{H}$ dissociation processes with simultaneous formation of $\mathrm{H}_{2}$ molecules have been identified in pm-Si:H and $\mathrm{Si}$ nanoparticles. ${ }^{9,13}$ In all cases, these processes lead to a peak in the DSC thermogram. Other authors have recently predicted that molecular diffusion is a significant process in amorphous silicon containing embedded nanocrystals (such as in pm-Si:H), ${ }^{14}$ and $a b$ initio simulations ${ }^{15}$ have reported the formation of $\mathrm{H}_{2}$ molecules in a-Si:H. However, until now, experiments have only reported evidence of significant amounts of $\mathrm{H}_{2}$ molecules in a-Si:H (Refs. 16 and 17) but not their diffusion.

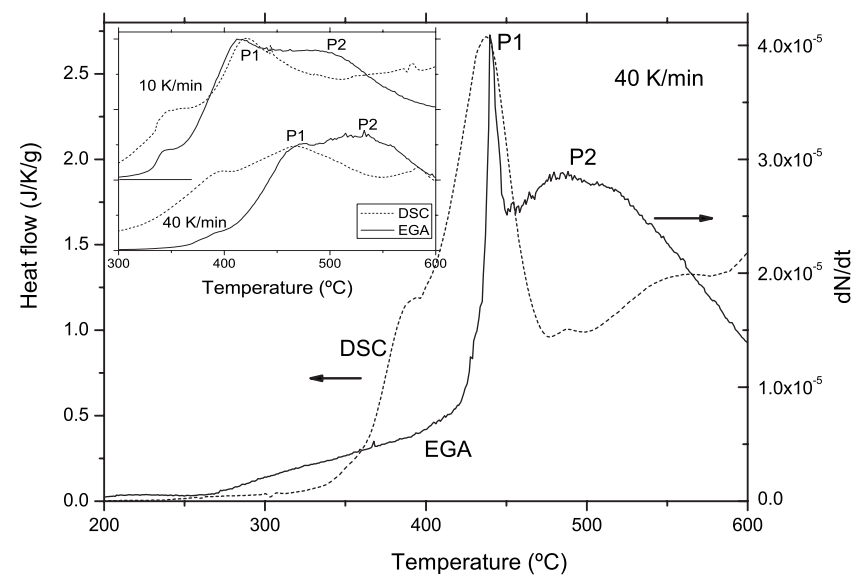

FIG. 1. DSC and EGA thermograms for a $2 \mu \mathrm{m}$ thick pm-Si:H thin film heated at $40 \mathrm{~K} / \mathrm{min}$. The inset shows DSC and EGA thermograms from Ref. 9 for a $5 \mu \mathrm{m}$ thick pm-Si:H film heated at 10 and $40 \mathrm{~K} / \mathrm{min}$ (Ref. 11). 
In this paper, we report on systematic EGA experiments on a series of device-quality pm-Si:H films of varying thicknesses, grown with the aim of demonstrating $\mathrm{H}_{2}$ molecular diffusion. The films were deposited on a glass substrate by plasma-enhanced chemical vapor deposition from a silane plasma highly diluted with hydrogen $(\sim 6 \%$ in volume $)$ at temperatures ranging from 175 to $225^{\circ} \mathrm{C}$. The EGA measurements were taken in vacuum $\left(\sim 10^{-6}\right.$ mbar $)$ with an MKS Spectra quadrupole (Micro Vision Plus). Samples were wrapped in platinum foil in contact with a thermocouple and placed inside a fused silica tube. DSC experiments were carried out with a Mettler Toledo calorimeter (DSC822e).

We performed a series of EGA experiments at different heating rates and film thicknesses to analyze desorption and diffusion processes. The results are plotted in Fig. 2. All the curves show the characteristic two-peak structure (P1-P2) already found for the thicker films (Fig. 1). In addition to the direct proof that P1 coincides with an exothermic peak in the DSC thermogram for the $2 \mu \mathrm{m}$ film, we can provide an indirect proof for the thinner films of the series where DSC experiments are much more difficult to perform. The lowtemperature desorption processes in a-Si:H are described by first-order reaction kinetics ${ }^{8,9,13,18}$ with rate constant, $K$, thermally activated according to

$$
K(T)=\frac{k T}{h} \exp \left(\frac{\Delta S}{k}\right) \exp \left(-\frac{E_{A}}{k T}\right)
$$

where $\Delta S$ is the activation entropy, $E_{A}$ is the activation enthalpy, and $h$ and $k$ are the Planck and Boltzmann constants, respectively. We determined $\Delta S$ and $E_{A}$ of peak P1 by means of the Kissinger plot described in Ref. 9 and drawn in the upper part of Fig. 3. The activation values of the five films were then compared with the values obtained by DSC experiments on thick pm-Si:H samples and a-Si:H nanoparticles $^{9}$ and EGA experiments on a-Si:H thin films ${ }^{18}$ (lower part of Fig. 3). A particular dehydrogenation process is related to an $\mathrm{H}$ state and is characterized by a linear relationship between its activation entropy and enthalpy that constitutes its signature. ${ }^{18}$ We see in Fig. 3 that, as expected from Fig. 1, the P1 points are close to the signature of $\mathrm{Si}-\mathrm{H}$ groups "at interfaces" determined by DSC. ${ }^{9}$ We can thus conclude that the EGA peak P1 is related to an exothermic process that takes place at the same temperature.

The experimental thermograms were fitted to a simple diffusion model. We consider that all $\mathrm{Si}-\mathrm{H}$ groups at interfaces are dissociated during the temperature interval of peak $\mathrm{P} 1$, and that the concentration of the diffusing species $\left(\mathrm{H}_{2}\right.$ molecules), $c(x, t)$, increases throughout the whole of the film volume. Consequently, $c(x, t)$ will be given by the diffusion equation

$$
\frac{\partial c}{\partial t}=D_{m}(t) \frac{\partial^{2} c}{\partial x^{2}}+g(t)
$$

where the formation of the $\mathrm{H}_{2}$ molecules is simulated by a Gaussian distribution, $g(t)$, centered at peak $\mathrm{P} 1$, and $D_{m}(t)$ is the diffusion coefficient of molecular $\mathrm{H}$,

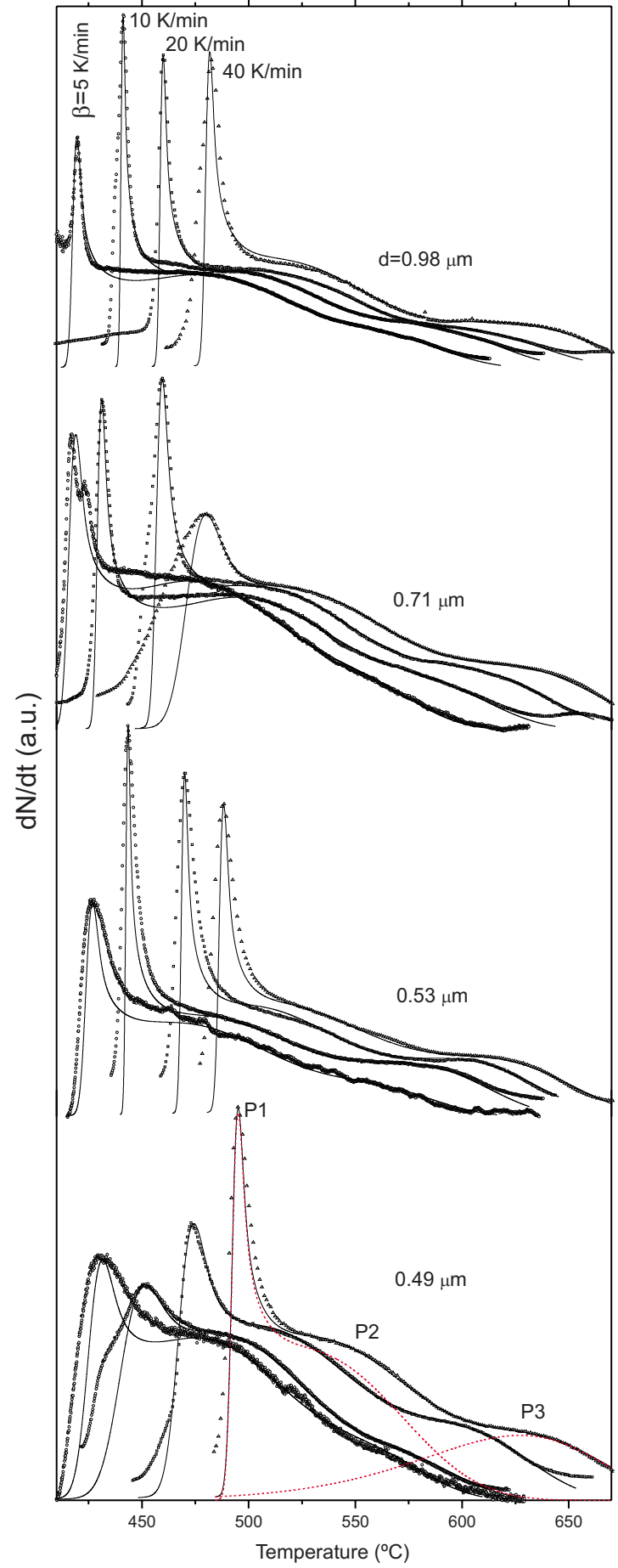

FIG. 2. (Color online) EGA thermograms obtained at different heating rates and film thicknesses (symbols) and fitted numerical solution of the diffusion equation (solid lines). The decomposition of the thermograms in two independent processes (one responsible for peaks P1 and P2 and a second responsible for peak P3) is indicated by the red dashed curves.

$$
D_{m}(t)=D_{m, 0} \exp \left[-E_{m} / k T(t)\right],
$$

where $E_{m}$ is the activation energy and $D_{m, 0}$ is the preexponential term and $T(t)=T_{0}+\beta t\left(T_{0}\right.$ is the initial temperature and $\beta$ is the constant heating rate). Equation (2) is 


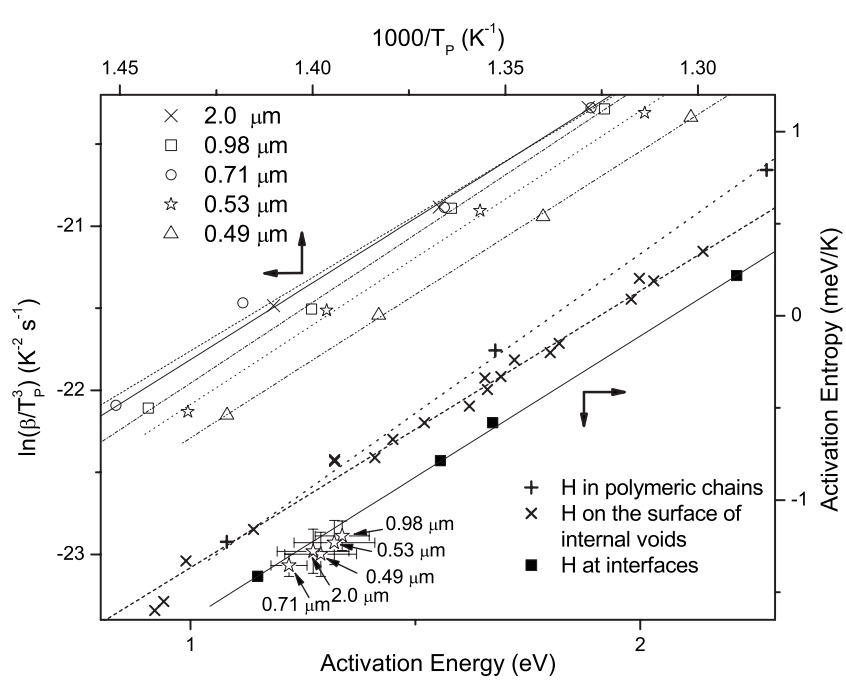

FIG. 3. Upper plot: Kissinger plot of peak P1 (Fig. 2) from which activation entropies and enthalpies were obtained. Lower plot: the activation entropy and enthalpy values of the films are plotted together with the characteristic signatures of several $\mathrm{Si}-\mathrm{H}$ groups determined from diverse hydrogenated silicon amorphous materials (Refs. 9, 18, and 19).

solved with the initial condition $c(x, 0)=0$ and the boundary conditions

$$
\partial x /\left.\partial t\right|_{x=0}=0 \text { and }\left.\quad c\right|_{x=d}=0,
$$

i.e., there is no hydrogen flow from the layer to the glass substrate (at $x=0$ ) and the hydrogen concentration at the free surface is negligible (at $x=d$ ). The latter condition is based on the extremely small sticking coefficient of molecular hydrogen in Si surfaces. ${ }^{20,21}$ This assumption is reinforced by the fact that there is not a significant delay between the heat released and the $\mathrm{H}_{2}$ desorption at peak P1. Once solved, the hydrogen evolution rate is given by

$$
\frac{d N}{d t}=-\frac{d}{d t} \int_{0}^{d} c(x, t) d x .
$$

In addition, to account for the smaller peak $\mathrm{P} 3$ appearing at a higher temperature, which is due to the $\mathrm{H}$ diffusion in atomic form ${ }^{8}$ the analytical solution given for this process in Ref. 22 has been added,

$$
\frac{d N}{d t}=\frac{2 c_{0} \pi}{d^{2}} D_{a}(t) \exp \left[-\frac{\pi}{d^{2}} \int_{0}^{t} D_{a}(z) d z\right],
$$

where $c_{0}$ is the initial concentration of $\mathrm{Si}-\mathrm{H}$ groups in the bulk of the film and $D_{a}(t)=D_{a, 0} \exp \left[-E_{a} / k T(t)\right]$ is the diffusion coefficient related to peak P3 with activation energy $E_{a}$ and pre-exponential term $D_{a, 0}$.

According to our model, the internal structure of EGA thermograms consists of the superposition of two independent desorption processes (dashed curves at the bottom of Fig. 2). The first process is due to the main $\mathrm{Si}-\mathrm{H}$ group present in pm-Si:H films ( $\mathrm{Si}-\mathrm{H}$ at interfaces) and has the characteristic two-peak structure. Although all $\mathrm{Si}-\mathrm{H}$ groups that dissociate at peak $\mathrm{P} 1$ form $\mathrm{H}_{2}$ molecules, only those

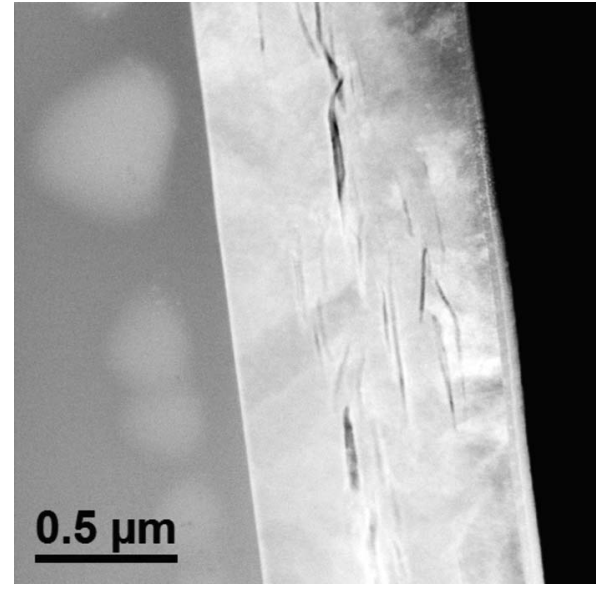

FIG. 4. TEM micrograph showing the transversal section of a pm-Si:H thin film after the dehydrogenation process (film thickness $=0.98 \mu \mathrm{m})$.

located near the free surface contribute almost instantaneously to the EGA signal at peak P1 whereas the rest of them contribute at the diffusion-delayed peak P2. Note that, due to their common origin, no free parameter can be used to fit the relative intensity of peaks P1 and P2. The second process is described by a single peak (P3) because, for this process, there is not a characteristic "dissociation temperature" that triggers the atomic diffusion of $\mathrm{H}$. Instead, $\mathrm{H}$ diffuses at any temperature in a trapping and releasing process that determines the concentration of mobile $\mathrm{H}$ atoms with respect to those trapped. ${ }^{7,23}$

The fitting process is quite simple. The parameters of the Gaussian function, $g(t)$, position and width, are approximately those of peak P1. An initial estimation of the molecular and atomic diffusion coefficients, $D_{m}$ and $D_{a}$, is obtained from the position of peaks $\mathrm{P} 2$ and $\mathrm{P} 3$, respectively. The diffusion coefficients are then fine tuned to obtain the best fit. From Fig. 2 it is apparent that this simple diffusion model provides a good description of the complete desorption process. From the fitting of the EGA thermograms we obtained the activation energy for peaks P2 and P3; for peak P3 this was $E_{a}=1.57 \pm 0.1 \mathrm{eV}$. Note that all fittings deliver practically the same activation energy. This value is in agreement with the approximate value of $\sim 1.5 \mathrm{eV}$ determined experimentally ${ }^{22,24-27}$ for the atomic diffusion in undoped a-Si:H, and confirms that peak P3 is due to atomic diffusion. For peak P2 the value we obtained for molecular diffusion was $E_{m}=0.99 \pm 0.1 \mathrm{eV}$. This value is consistent with nuclear-magnetic-resonance (NMR) experiments. ${ }^{16}$ Indeed, it has been observed that molecular $\mathrm{H}$ is located in the amorphous equivalent of tetragonal $T$ sites and that the jumps from $T$ site to $T$ site require an activation energy of approximately $1 \mathrm{eV}$. This activation energy is slightly higher than that observed in crystalline silicon $(0.78-0.91 \mathrm{eV}){ }^{28,29}$

With regard to the pre-exponential term $D_{m, 0}$, this varies over 1 order of magnitude. We attribute this dispersion to the fact that, in pm-Si:H films, the sudden formation of a large amount of $\mathrm{H}_{2}$ molecules (peak P1) results in cracking. ${ }^{30} \mathrm{In}$ Fig. 4 one can observe the formation of these cracks in a $0.98 \mu \mathrm{m}$ thick film after dehydrogenation. As a consequence 
of the cracking, the diffusion length is not equal to film thickness and an "effective diffusion length," related to the average distance between cracks, is more appropriate to describe the results. The existence of a nearly common effective diffusion length for all the films is reinforced by the fact that the peak temperature of P3 (without any doubt controlled by diffusion) is independent of the film thickness.

In conclusion, we have reported on a series of systematic experiments designed to demonstrate that hydrogen diffuses in pm-Si:H mainly in molecular form. Several relevant results from the analysis of EGA measurements on samples with different thickness point out to the same conclusion, namely: (i) the signature of peak P1 confirms that it is related to $\mathrm{Si}-\mathrm{H}$ bond breaking and it corresponds to a particular $\mathrm{H}$ state of pm-Si (Ref. 9). Since this signature is close to that of $\mathrm{Si}-\mathrm{H}$ on the surface of voids, ${ }^{18}$ the bond-breaking process must be very similar (i.e., it entails simultaneous formation of a $\mathrm{H}_{2}$ molecule); (ii) on the contrary, peak P2 is much broader than expected for the simple reaction kinetics governing the low-temperature dehydrogenation processes in a-Si:H (usually first-order kinetics). Its full width at half maximum would deliver an unrealistic activation energy of $0.5 \mathrm{eV}$; (iii) the complete EGA spectrum with three peaks can be fitted to a simple diffusion model where it is assumed that only two species diffuse, molecular and atomic hydro- gen. The characteristic two-peak structure for the molecular process with its sharp low-temperature peak is unveiled; (iv) the diffusion model would not lead to two distinct EGA peaks if one single species was diffusing. So, detection of P1 and P2 peaks indicates that two different species diffuse; (v) from the model fitting, the activation energies for molecular and atomic hydrogen diffusion are obtained. The first activation energy agrees with NMR experiments while the second one coincides with the well-known value of the activation energy of atomic hydrogen diffusion.

Molecular diffusion involves breaking $\mathrm{Si}-\mathrm{H}$ groups that are presumably located at the interfaces between nanocrystallites and the amorphous matrix of pm-Si:H. Consequently, if active, this mechanism would be of secondary importance in monophasic a-Si:H. However, we think that it could be the main diffusion mechanism in microcrystalline silicon ( $\mu \mathrm{c}-\mathrm{Si}: \mathrm{H})$. In fact, EGA thermograms of this material exhibit the two-peak structure analyzed here. ${ }^{30}$

This work was partially funded by the Spanish Ministerio de Educacin y Ciencia (MAT2006-11144 Project and SB2006-0062 postdoctoral Contract) and by the Generalitat de Catalunya (Contract No. 2005SGR-00666). The authors are indebted to J. Arbiol from the Serveis Científico-Tècnics (University of Barcelona) for the TEM micrograph. *fatiha.kail@udg.cat

${ }^{1}$ R. Street, Hydrogenated Amorphous Silicon (Cambridge University Press, New York, 1991).

${ }^{2}$ D. L. Staebler and C. R. Wronski, Appl. Phys. Lett. 31, 292 (1977).

${ }^{3}$ H. M. Branz, Phys. Rev. B 59, 5498 (1999).

${ }^{4}$ O. Saadane, S. Lebib, A. V. Kharchenko, C. Longeaud, and P. Roca i Cabarrocas, J. Appl. Phys. 93, 9371 (2003).

${ }^{5}$ C. Longeaud, J. P. Kleider, P. Roca i Cabarrocas, S. Hamma, R. Meaudre, and M. Meaudre, J. Non-Cryst. Solids 227-230, 96 (1998).

${ }^{6}$ P. St'ahel, S. Hamma, P. Sládek, and P. Roca i Cabarrocas, J. Non-Cryst. Solids 227-230, 276 (1998).

${ }^{7}$ W. Beyer, Sol. Energy Mater. Sol. Cells 78, 235 (2003).

${ }^{8}$ W. Beyer, Physica B 170, 105 (1991).

${ }^{9}$ P. Roura, J. Farjas, C. Rath, J. Serra-Miralles, E. Bertran, and P. Roca i Cabarrocas, Phys. Rev. B 73, 085203 (2006).

${ }^{10}$ P. Roura, J. Farjas, and P. Roca i Cabarrocas, Thin Solid Films 517, 6239 (2009).

${ }^{11}$ Although the coincidence of peak P1 here highlighted passed unnoticed in Ref. 9, the main conclusion of that paper remains unchanged

${ }^{12}$ D. Das, J. Farjas, P. Roura, G. Viera, and E. Bertran, Appl. Phys. Lett. 79, 3705 (2001).

${ }^{13}$ J. Farjas, D. Das, J. Fort, P. Roura, and E. Bertran, Phys. Rev. B 65, 115403 (2002).

${ }^{14}$ B. C. Pan and R. Biswas, J. Non-Cryst. Solids 333, 44 (2004).

${ }^{15}$ S. Chakraborty and D. A. Drabold, Phys. Rev. B 79, 115214
(2009).

${ }^{16}$ P. A. Fedders, D. J. Leopold, P. H. Chan, R. Borzi, and R. E. Norberg, Phys. Rev. Lett. 85, 401 (2000).

${ }^{17}$ T. Su, S. Chen, P. C. Taylor, R. S. Crandall, and A. H. Mahan, Phys. Rev. B 62, 12849 (2000).

${ }^{18}$ Y. L. Khait, R. Weil, R. Beserman, W. Beyer, and H. Wagner, Phys. Rev. B 42, 9000 (1990).

${ }^{19}$ J. Costa, J. Fort, J. Sunol, P. Roura, G. Viera, and E. Bertran, in Hydrogen in Semiconductors and Metals, edited by N. H. Nickel, W. B. Jackson, R. C. Bowman, and R. G. Leisure (Mater. Res. Soc., Warrendale, 1998), Vol. 513, pp. 427-432.

${ }^{20}$ M. Dürr and U. Höfer, Surf. Sci. Rep. 61, 465 (2006).

${ }^{21}$ J. T. Law, J. Chem. Phys. 30, 1568 (1959).

${ }^{22}$ W. Beyer and H. Wagner, J. Appl. Phys. 53, 8745 (1982).

${ }^{23}$ A. J. Franz, M. Mavrikakis, and J. L. Gland, Phys. Rev. B 57, 3927 (1998).

${ }^{24}$ D. E. Carlson and C. W. Magee, Appl. Phys. Lett. 33, 81 (1978).

${ }^{25}$ K. Zellama, P. Germain, S. Squelard, B. Bourdon, J. Fontenille, and R. Danielou, Phys. Rev. B 23, 6648 (1981).

${ }^{26}$ W. B. Jackson and C. C. Tsai, Phys. Rev. B 45, 6564 (1992).

${ }^{27}$ I. An, Y. M. Li, C. R. Wronski, and R. W. Collins, Phys. Rev. B 48, 4464 (1993).

${ }^{28}$ N. M. Johnson and C. Herring, Phys. Rev. B 43, 14297 (1991).

${ }^{29}$ V. P. Markevich and M. Suezawa, J. Appl. Phys. 83, 2988 (1998).

${ }^{30}$ S. Lebib and P. Roca i Cabarrocas, Eur. Phys. J. Appl. Phys. 26, 17 (2004) 